\title{
Dynamic buckling of laminated composite stringer stiffened CFRP panels under axial compression
}

\author{
Mouhat Ouadia ${ }^{1}$, Khamlichi Abdellatif ${ }^{2}$, Hasnae Boubel ${ }^{1}$, Oumnia Elmrabet ${ }^{1}$, Mohamed Rougui $^{1}$, El Mehdi Echebba ${ }^{1}$ \\ and Ahmed El Bouhmidi ${ }^{1}$ \\ ${ }^{1}$ Mohammed V University, Structure LGCE l'EST, Salé -CED de l'EMI, Rabat, MOROCCO \\ ${ }^{2}$ Abdelmalek Essaadi University, Communications Systems and Detection Laboratory, Tetouan, MOROCCO
}

\begin{abstract}
In this work, the dynamic buckling of stiffened panels is evolved numerically through a nonlinear incremental expression through making use of a specific time integration procedure via the finite element software program. the buckling and post-buckling behaviours of hat-stringer-stiffened composite curved panel under axial compression load .Dynamic buckling is extracted from the curve abandoning the very last shortening as a characteristic of time while the shape is subjected with the aid of a square compression pulse movement carried out inside the axial direction. The duration of the heart beat and the amplitude of curvature of decreasing of the cloth inside the band tormented by the warmth, the dynamic buckling motion, are constant. The method approach was proposed to predict the dynamic buckling load of curved panel. Finite element analysis was used to investigate these tests and the FE models were performed by ABAQUS.Approach to determine the reliability of the stiffened panel in dynamic buckling state.
\end{abstract}

Keywords: Dynamic buckling, stiffened panels, axial compression, finite element method, reliability.

\section{Introduction}

Stiffened composite curved panel is a model structure widely used in aeronautic industry and spacecraft in the last few years, in the industry efficiency and performance of the structure is the essential objective for the design which requires insistence on the need for strong and light materials. So that the resistance must be high for this in utilised the reinforced polymer fibres for this we find the Stiffened composite curved panel is in several fields of application. it is necessary for the design to study the stability and buckling of the stiff composite panel of the many factors that can affect the buckling characteristics and the post-buckling, Many experimental tests and numerical calculations have been realized to study the behaviour of static and dynamic buckling several researchers who are published in this axis on the static buckling but we are interested in this work by the dynamic buckling.

During the last two decades many articles studied stiffened curved panels under axial compression
loading[1-3].Recently, the project that has been the focus of attention in previous years is the project POSICOES and COCO MAT [4] ,Both supported by the European Commission, to study the behavior of composite panels stiffened by all the buckling and post buckling more and more of attention is given to buckling and The aerospace industry has asked to reduce development and operating costs by $20 \%$ and $50 \%$ in the short and long term. There is a difficulty in preaching the critical load of buckling and the ultimate load by the classical methods. Von Karman et al. [5] a proposed approach "effective width" for the optimization this has been modified by several carters among these loaders Wang Zhenming [6]. There are several experiences of stiffened curved panels under axial compression loading but most are forming I-stiffened panels [7]. And T-stiffened panel [8].

Determination of relative response for the boring load parameters by equating the equations of the motion and trace the selected axial displacement curve as a function of time. The specific value of the charge from this change 
coincides with the critical value associated with dynamic buckling according to the Budiansky-Roth criterion [9] (Budiansky and Roth, 1962). This criterion seems more practical in the study of dynamic buckling of thin structures.

In the aeronautical and aerospace industries the lifespan of the aircraft is civil or military, generally the aircraft that exceeds 20 years of service or not even, so the buckling performance of stiffened composite panels must also

be

a big concern for the airplane designer. Scientists now focus on the dynamic buckling that was often ignored composite panels. This article discusses the nonlinear method and dynamic buckling of the hat-stringerstiffened Composite flat panel subjected to axial compression and made a reliability analysis using the Matlab Monte Carlo method to calculate the probability of failure.

\section{Panel description}

The stiffened composite panel is composed of three stiffeners. The nominal length and the nominal width of the panel are $952 \mathrm{~mm}$ and $757.5 \mathrm{~mm}$. The gap between two stiffeners is $252.5 \mathrm{~mm}$. The two terminals of the panel were recessed ensuring a uniform distributed load calculation. The mechanical properties of the material are defined in Table 1. The layout of the laminate at each zone of the panel is reported in Table 2 and specified graphically in the stiffeners are L-shaped and have the constant

$$
\text { thickness } \mathrm{t}_{\mathrm{w}}=2.95 \mathrm{~mm} \text {, }
$$

height $\mathrm{h}_{\mathrm{w}}=64 \mathrm{~mm}$, the thickness of the flange $t_{f}=4.3 \mathrm{~mm}$ and the height of the flange $b_{f}=12 \mathrm{~mm}$. Specified graphically in Fig. 1,Nominal ply thickness is $0.185 \mathrm{~mm}$.

In Fig.2. Shows the boundary conditions the configuration of the stiffened panel.

By indicating $\mathrm{u}_{\square}$ the displacement coordinates and $\theta_{\square}$ rotations, the boundary conditions examined in the software abaqus are as follows. The lateral ends have the following boundary conditions $u_{x}=\theta_{x}=\theta_{z}=0$. The border $\mathrm{Z}=0$ is supposed to be perfectly wet $\mathrm{u}_{\mathrm{x}}=\mathrm{u}_{\mathrm{y}}=\mathrm{u}_{\mathrm{z}}=\theta_{\mathrm{x}}=\theta_{\mathrm{y}}=\theta_{\mathrm{z}}=0$, as the uniformly distributed load $\mathrm{P}_{\mathrm{z}}$ is applied to the edge $\mathrm{Z}=\mathrm{a}$ with rigid boundary conditions on the side $\mathrm{u}_{\mathrm{x}}=\theta_{\mathrm{x}}=\theta_{\mathrm{z}}=0$.

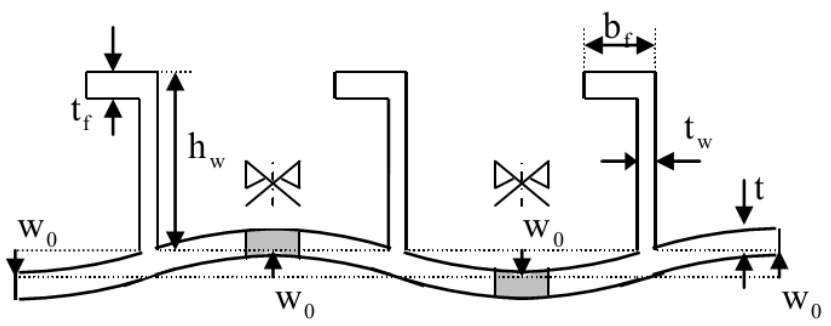

Fig. 1. The initial configuration in of the stiffener panel

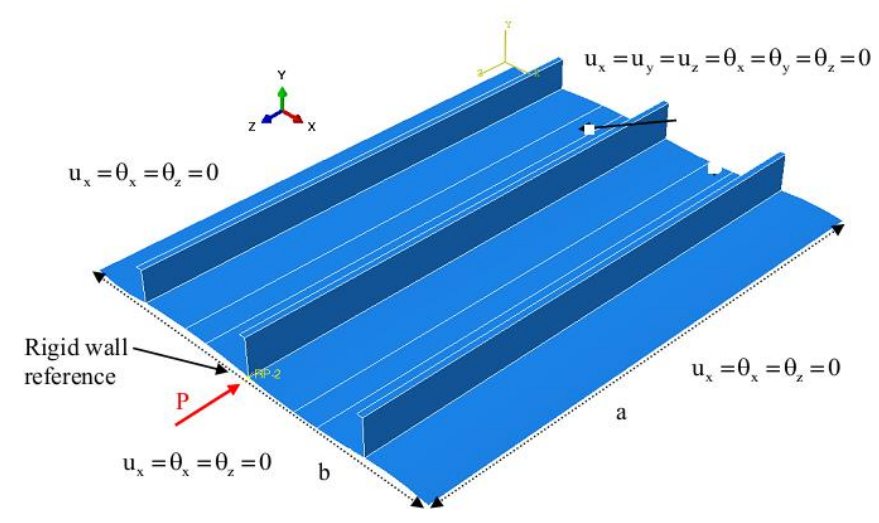

Fig.2. Geometry of panel and boundary condition

Table 1. Mechanical properties

\begin{tabular}{|c|c|c|c|}
\hline \multicolumn{2}{|c|}{ Stiffness } & \multicolumn{2}{c|}{ strength } \\
\hline $\mathrm{E}_{1}$ & $141 \mathrm{GPa}$ & $\mathrm{X}_{\mathrm{T}}$ & $2700 \mathrm{MPa}$ \\
\hline $\mathrm{E}_{2}$ & $8.85 \mathrm{GPa}$ & $\mathrm{X}_{\mathrm{C}}$ & $1600 \mathrm{MPa}$ \\
\hline $\mathrm{G}_{12}=\mathrm{G}_{13}$ & $4.57 \mathrm{GPa}$ & $\mathrm{Y}_{\mathrm{T}}$ & $88 \mathrm{MPa}$ \\
\hline $\mathrm{G}_{23}$ & $4.2 \mathrm{GPa}$ & $\mathrm{Y}_{\mathrm{C}}$ & $271 \mathrm{MPa}$ \\
\hline$v_{12}$ & 0.33 & $\mathrm{~S}$ & $143 \mathrm{MPa}$ \\
\hline
\end{tabular}


Table 2.Lay-up definition

\begin{tabular}{|c|c|}
\hline Skin & $t_{w}\left[\begin{array}{l}45 /-45 / 90 / 90 / 0 / 90 / 0 \\
/ 90 / 0 / 90 / 90 /-45 / 45\end{array}\right]$ \\
\hline Flange & $t_{f}[45 /-45 / 0 / 90 / 90 / 0 /-45 / 45]$ \\
\hline
\end{tabular}

\section{Buckling analysis of stiffened panels}

\subsection{Composite materials}

Composite means in the term composite materials either two or more materials are macerated on a macroscopic scale to form a third useful material. Different materials can be mixing at the macroscopic scale as in the metal alloy, but the resulting materials are, for all practical purposes, macroscopically homogeneous, that is to say that the composite cannot be distinguished with the naked eye and act essentially together.

\subsection{Classifications of composite materials}

- A laminate consists of a stack of monolayers each a proper orientation with respect to a common frame of reference layers and designated as the laminate reference frame.

- Particulate Composites consist of a matrix reinforced by a dispersed phase in form of particles.

- Fibrous composite materials that consist of fibres in a matrix.

- Combination of some or all of the first three types.

\subsection{Stiffness and strength of a lamina}

The orientation of the fibres can be randomly in the material; in reality the fibres in the preferred directions can be oriented in expectation of the highest stresses. Such a material is said to be anisotropic, which is different from their physical properties (elasticity moduli, Poisson coefficients, thermal conductivity, etc.) which have different values depending on the spatial orientation of the physical body.

The microscopic level, the properties of these composites are found by the orientation and distribution of the fibres, as well as by the properties of the fibre and matrix substances.

Consider a region of unit dimensions in a material type, which material contains a volume fraction, $V_{f}$ we chose all orientations in a single direction, with the volume fraction of the matrix is written by this following formula:

$V_{m}=1-V_{f}$

This place can be made in conglomerate all the fibers together, dismissal the matrix to occupy the remaining volume. If a stress $\sigma_{l}$ applied along the direction of the fibre, the phases of the fibre and the matrix proceed in similar to support the load. In these parallel bonds the constraints in each period must be the same, so that the strain $\varepsilon_{l}$ in the direction of the fibre can be written in this way as:

$\varepsilon_{l}=\varepsilon_{f}=\varepsilon_{m}$

With: the indices $\mathrm{L}$ and $\mathrm{m}$ denote the lamina and the fibres and the matrix respectively).

For the balance of the total charge in the composite material are added the forces in each phase.

Since the forces in each period are the period constraints times the area (here theoretically equal to the volume fraction), we have:

$\sigma_{t}=\sigma_{f} V_{f}+\sigma_{m} V_{m}=E_{f} \varepsilon_{l} V_{f}+E_{m} \varepsilon_{l} V_{m}$

The stiffness in the direction of the fibres is found by dividing the stress by the strain:

$E=\frac{\sigma_{l}}{\varepsilon_{l}}=E_{f} V_{f}+E_{m} V_{m}$

With: E is the longitudinal Young's modulus.

For the prediction of the mixtures of the global module using this relation which was according to the terms of the modules of hashes which constitutes the volume fractions. 
Rule assessments of mixtures for force flow along lines similar to those for rigidity. For example, consider a unidirectional reinforced composite that is stretched to the value at which the fibre begins to break. If the matrix is more ductile than the fibres, then the ultimate tensile strength of the lamina in the equation (3) changed to:

$$
\sigma_{t}^{u}=\sigma_{f}^{u} V_{f}+\sigma_{m}^{f}\left(1-V_{f}\right)
$$

When the power $u$ shows an extreme value, and $\sigma_{m}^{f}$ is the stress of the matrix when the fibres break as in fig. 3 . It is visible that if the volume fraction of the fibre is not strong, the attitude of the lamina is examined by the matrix.

Mathematically expressed by this following relation:

$$
\sigma_{l}^{u}=\sigma_{m}^{u}\left(1-V_{f}\right)
$$

if it is assumed that the lamina is to be used in a practical application, the minimum fraction of fibre volume is deduced this fraction must be added to the matrix, this formula obtained by solving the equations respectively (5) and (6).

Mathematically expressed by this following relation:

$V_{\text {min }}=\frac{\sigma_{m}^{u}-\sigma_{m}^{f}}{\sigma_{f}^{u}+\sigma_{m}^{u}-\sigma_{m}^{f}}$

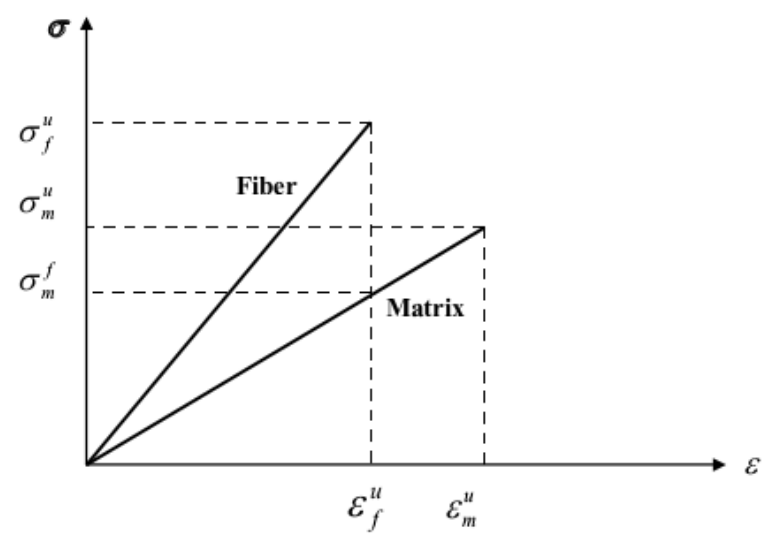

Fig.3.Stress-strain for fiber and matrix

\subsection{Nonlinear dynamic buckling analysis}

For the dynamic buckling test, the explicit dynamic instruction makes it possible to perform the whole small increment time determination to find a working approximation to the resolution of the problem. This procedure is based on the time integration rule. The increment calculation method is a bit expensive compared to the implicit forward dynamic integration dynamic analysis instruction does not involve any inversion of a linear system. The explicit federation dissimilarity manipulator satisfies the dynamic equilibrium equations at the origin of the time increment $t$ , the increments calculated at that time are used $\mathrm{t}+\Delta \mathrm{t} / 2$ to advance the time-to-time solution $\mathrm{t}+\Delta \mathrm{t}$ and the ontime move solution.. Generously the principle of preliminary kinematics of an arbitrary time step in acceleration and velocity completions.

Giving the kinematics state at the beginning of a given time step in terms of the acceleration $\ddot{\mathrm{u}}_{n}$ and the velocity $\dot{\mathrm{u}}_{\mathrm{n}-1 / 2}$, the equations of motion are integrated using the explicit central-difference integration rule according to

$$
\begin{aligned}
& \dot{\mathrm{u}}_{\mathrm{n}+1 / 2}=\dot{\mathrm{u}}_{\mathrm{n}-1 / 2}+\frac{\Delta \mathrm{t}_{\mathrm{n}+1}+\Delta \mathrm{t}_{\mathrm{n}}}{2} \ddot{\mathrm{u}}_{\mathrm{n}} \\
& \mathrm{u}_{\mathrm{n}+1}=\mathrm{u}_{\mathrm{n}}+\Delta \mathrm{t}_{\mathrm{n}+1} \dot{\mathrm{u}}_{\mathrm{n}+1 / 2}
\end{aligned}
$$

With $\mathrm{n}$ refers to the step number.

\section{Reliability analysis of composite stringer stiffened panels}

To complete a reliability analysis, an analytical model giving an explicit abstract of the problem is developed. This is completed by selecting appropriate application points on the deep division of explored variables according to an experimental design scale (DOE). This table is generated by setting distinguished levels of intermediate factors. Beginning with the objectives received from the simulation generated for these particular points, a model is originated in order to affirm the state function in an explicit form over the whole domain used for the regression. A rectangle polynomial response surface abbreviation is provided. 
The variability of the dynamic buckling load is assumed to start from the following three constituent sources which include the magnitude of the geometric imperfection, the Young's modulus, and the square pulse period. All referees geometric and mechanical parameters.

Keeping only these three active variables, the buckling load can be expressed explicitly as

$\mathrm{P}_{\mathrm{cr}}=\mathrm{P}_{\mathrm{cr}}\left(\mathrm{w}_{0}, \mathrm{E}, \mathrm{T}\right)$

In this anticipation which makes it possible to focus on the fundamental effects governing the problem of dynamic buckling of stiffened panels, the derivation of a predictive model is considered under the adaptation of a rectangle polynomial response surface. $\mathrm{P}_{\mathrm{cr}}$.

\section{Results and discussion}

Table.3. gives the levels of the three parameters selected to perform the reliability analysis. The column $\mathrm{T}$ corresponds to the following three values: $T=0.25 T_{0}, 0.5 T_{0}, 0.75 T_{0}, T_{0}$ with $\mathrm{T}_{0}=12.45 \mathrm{~ms}$

\begin{tabular}{|c|c|c|c|}
\hline $\begin{array}{c}\text { Parameter } \\
\text { level }\end{array}$ & $w_{0}(\mathrm{~mm})$ & $E(G P a)$ & $T(m s)$ \\
\hline Low & 2 & 64 & 6.12 \\
\hline medium & 3 & 66.75 & 9.28 \\
\hline High & 4 & 69.5 & 12.45 \\
\hline
\end{tabular}

Table 3. Levels of factors considered in the reliability analysis

Table 2. Is used to construct a complete factorial table consisting of 27 combinations involving the levels specified.
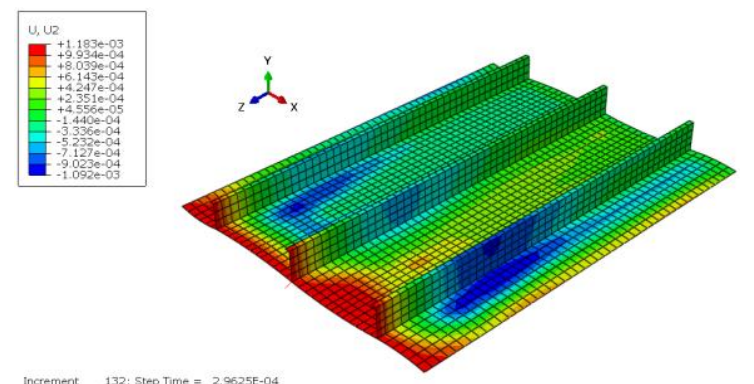

(a)
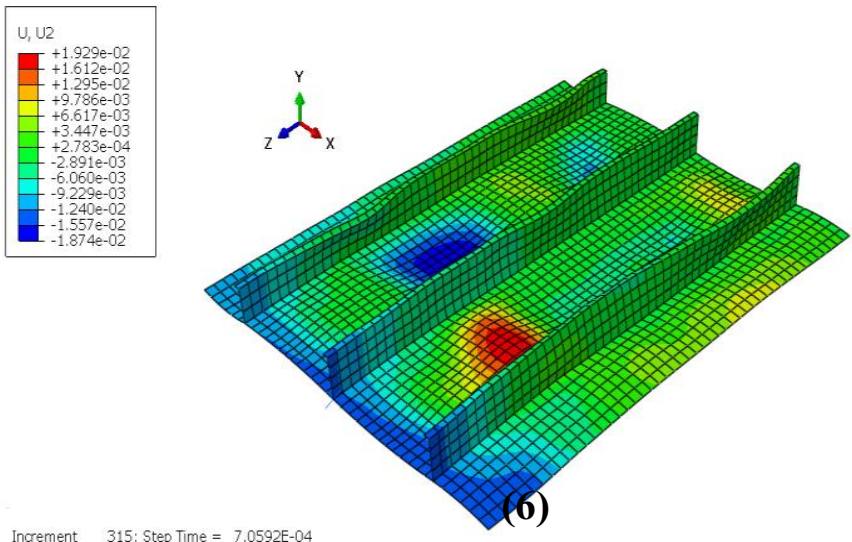

Increment 315: Step Time $=7.0592 E-04$
Primary Var: U, U2
Deformed Var: $U$ Deformation Scale Factor: $+1.000++00$

(b)
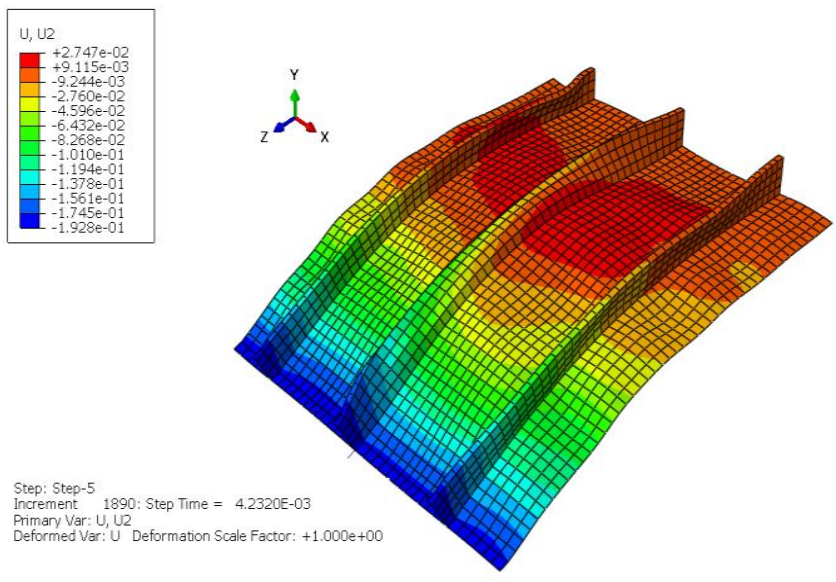

(c)

Fig.4. Three sequences of deformation of the stiffened panel in the form of the iso-values of the transverse displacement.

In order to obtain a response surface that represents the dynamic buckling critical load in an explicit form with coefficients of the same order of magnitude, the factors of Table .3 are dimensioned by asking:

$\bar{w}_{0}=w_{0} / w_{0, \max }, \quad \bar{E}=E / E_{\max }$ et $\bar{T}=T / T_{\max }$

The dynamic buckling critical stress and coefficients of this polynomial are obtained by the regstats command of Matlab and the response surface polynomial of the computed system is:

$$
\begin{aligned}
& \sigma_{\mathrm{cr}}(\mathrm{MPa})=-5.12+19.25 \tilde{\mathrm{w}}_{0}-4.51 \tilde{\mathrm{E}}- \\
& 0.25 \tilde{\mathrm{T}}-1.84 \tilde{\mathrm{w}}_{0} \tilde{\mathrm{E}}+1.24 \tilde{\mathrm{w}}_{0} \tilde{\mathrm{T}}-0.961 \tilde{\mathrm{E}} \tilde{\mathrm{T}} \\
& -8.24 \tilde{\mathrm{w}}_{0}^{2}+7.58 \tilde{\mathrm{E}}^{2}+3.65 \tilde{\mathrm{T}}^{2}
\end{aligned}
$$


The interpolation defined by equation (11) has an excellent coefficient of determination $R^{2}=98.13 \%$. It should be noted that even a linear polynomial regression model also gives a very good coefficient of determination that is worth $R^{2}=99.3 \%$.

\subsection{Analysis of variance}

The analysis of variance performed on the results presented in Table .3 using the Matlab anovan command we can notice that the factors and their interactions correctly explain the variability of the dynamic buckling load because the residual error does not exceed. If one works with a linear model then the factors explain by themselves the results with an error limited to $3.4 \%$.

These results are very revealing of the phenomenology of the dynamic buckling which appears in this way as a phenomenon essentially governed by the duration of the impulse loading.

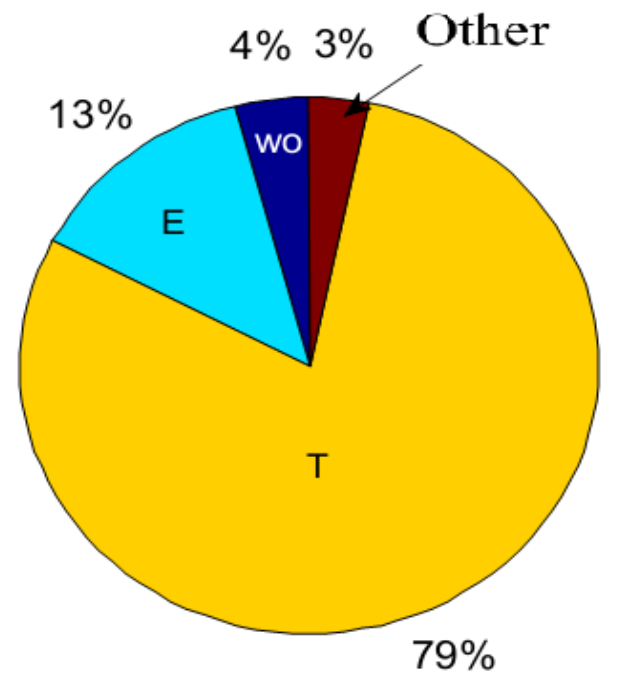

Fig 5. Relative influence of the factors describing the variability of the dynamic buckling critical load; "Other" includes interactions and error

The Fig. 5 gives the relative percentage of the influence of each factor on the variability of the dynamic buckling critical load.

\section{Conclusions}

This paper discusses dynamic buckling on composite stiffened panels when exposed to axial compression in the manner of a hat and axially reinforced. To obtain a variety of dynamic buckling, panels with different amplitude of curvatures, with different panel thicknesses these models based on ABAQUS software. This allowed the derivation of a surface response representation of dynamic buckling load variations.

\section{References}

1. E. Gal ,R. Levy ,H. Abramovich , P.Pavsner . Buckling analysis of composite panels.Compos Struct J.E 73,17985 (2006).

2. C.Bisagni, C.Potito. An experimental investigation into the buckling and post-buckling of CFRP shells under combined axial and torsion loading. Compos Struct J.E 60,391-402 (2003).

3. R.Zimmermann, H.Klein , A.Kling. Buckling and postbuckling of stringer stiffened fibre composite curved panels - tests and computations. Compos Struct ,J E 73 ,150-61 (2006).

4. R.Zimmermann , R.Rolfes . Posicoss-improved postbuckling simulation fordesign of fiber composite stiffened fuselage structures. Compos Structure, J.E 73,4171,(2006).

5. T.Von Karman , E.E.Sechler , L.H.Donnel . The strength of thin plates in compression. ASME Appl Mech Trans,J.E 54 (1932).

6. X.M.Wang, W.Cao, C.H.Deng. Experimental and numerical analysis for the post-buckling behavior of stiffened composite panels with impact damage. Compos Struct 2,J.E 133 (2015).

7. F.Caputo , R.Esposito, P.Perugini, D.Santoro. Numerical-experimental investigation on post-buckled stiffened composite panels. Compos Struct ,J.E 55,34757 (2002).

8. L.Lanzi , V.Giavotto . Post-buckling optimization of composite stiffened panels: computations and experiments. Compos Struct ,J.E 73,208-20 (2006).

9. B.Budiansky, J.W.Hutchinson . Dynamic buckling of imperfection-sensitive structures Proc. 11th Int. Congress of Applied Mechanics (Berlin: Springer) ,J.E.13,636-651, (1964) 